\title{
Melanocortin 4 receptor signals at the neuronal primary cilium to control food intake and body weight
}

\author{
Yi Wang, ${ }^{1}$ Adelaide Bernard, ${ }^{1}$ Fanny Comblain, ${ }^{1}$ Xinyu Yue, ${ }^{1}$ Christophe Paillart, ${ }^{1}$ Sumei Zhang, ${ }^{1}$ Jeremy F. Reiter, ${ }^{2,3}$ \\ and Christian Vaisse ${ }^{1}$ \\ 'Department of Medicine and The Diabetes Center and 'Department of Biochemistry and Biophysics, Cardiovascular Research Institute, UCSF, San Francisco, California, USA. \\ ${ }^{3}$ Chan Zuckerberg Biohub, San Francisco, California, USA.
}

\begin{abstract}
The melanocortin 4 receptor (MC4R) plays a critical role in the long-term regulation of energy homeostasis, and mutations in the MC4R are the most common cause of monogenic obesity. However, the precise molecular and cellular mechanisms underlying the maintenance of energy balance within MC4R-expressing neurons are unknown. We recently reported that the MC4R localizes to the primary cilium, a cellular organelle that allows for partitioning of incoming cellular signals, raising the question of whether the MC4R functions in this organelle. Here, using mouse genetic approaches, we found that cilia were required specifically on MC4R-expressing neurons for the control of energy homeostasis. Moreover, these cilia were critical for pharmacological activators of the MC4R to exert an anorexigenic effect. The MC4R is expressed in multiple brain regions. Using targeted deletion of primary cilia, we found that cilia in the paraventricular nucleus of the hypothalamus (PVN) were essential to restrict food intake. MC4R activation increased adenylyl cyclase (AC) activity. As with the removal of cilia, inhibition of AC activity in the cilia of MC4R-expressing neurons of the PVN caused hyperphagia and obesity. Thus, the MC4R signaled via PVN neuron cilia to control food intake and body weight. We propose that defects in ciliary localization of the MC4R cause obesity in human inherited obesity syndromes and ciliopathies.
\end{abstract}

\section{Introduction}

Most mammalian cells, including neurons, possess a single, immotile primary cilium, an organelle that transduces select signals (1). Defects in the genesis or function of primary cilia cause a range of overlapping human diseases, collectively termed ciliopathies (2). Several ciliopathies, such as Bardet-Biedl syndrome and Alström syndrome, cause obesity, and mutations in genes encoding ciliary proteins, such as CEP19, ANKRD26, and ADCY3, cause nonsyndromic obesity in mice and humans (3-6). Although the mechanisms underlying a number of cilia-associated phenotypes, such as polycystic kidney disease or retinal degeneration, have been at least partly elucidated, how ciliary dysfunction leads to obesity remains poorly understood $(7,8)$. Since primary cilia are essential for embryonic development, in particular through their critical role in Hedgehog signaling $(9,10)$, it has been hypothesized that obesity could result from perturbations in the development of cells and pathways implicated in the regulation of energy homeostasis. However, ubiquitous ablation of primary cilia in adult mice or in neurons also leads to obesity (11). This finding indicates that neuronal primary cilia are post-developmentally required for the function of one or more signaling pathways implicated in the regulation of energy homeostasis.

Authorship note: YW, AB, and FC contributed equally to this work

Conflict of interest: The authors have declared that no conflict of interest exists.

Copyright: (5) 2021, American Society for Clinical Investigation.

Submitted: July 13, 2020; Accepted: March 18, 2021; Published: May 3, 2021.

Reference information: J Clin Invest. 2021;131(9):e142064.

https://doi.org/10.1172/JCl142064.
We recently demonstrated that the melanocortin 4 receptor (MC4R) localizes to neuronal primary cilia in vivo (12). MC4R is a GPCR essential for long-term regulation of energy homeostasis (13). In humans, polymorphisms at the MC4R locus are tightly associated with obesity in GWAS (14), heterozygous mutations in the $M C 4 R$-coding sequence are the most common monogenic cause of severe obesity, and individuals with homozygous null mutations have severe, early-onset obesity (15-17). In mice, deletion of $M c 4 r$ causes hyperphagia and severe obesity (18). The MC4R is found in a number of neuronal populations, but its expression in the paraventricular nucleus of the hypothalamus (PVN) is both necessary and sufficient for the regulation of food intake and body weight (19).

MC4R activity is regulated by 2 endogenous ligands, an anorexigenic agonist, $\alpha$ melanocyte-stimulating hormone $(\alpha-\mathrm{MSH})$, and an orexigenic antagonist/inverse agonist, agouti-related peptide (AgRP). These neuropeptides are produced by neurons of the arcuate nucleus of the hypothalamus (ARC) under the control of the adipocyte-secreted hormone leptin (13). As leptin levels are proportional to fat mass (20), the observation that the MC4R can localize to neuronal primary cilia suggests that signaling from the primary cilia of MC4R-expressing neurons is a rate-limiting step in the sensing of energy stores and conditional modulation of energy intake.

Here, we used a combination of genetic and pharmacological approaches to demonstrate that primary cilia are required for ligand-dependent activation of the MC4R and long-term regulation of energy homeostasis by MC4R-expressing PVN neurons, and that altered MC4R ciliary function can underlie ciliopathy-associated obesity. 

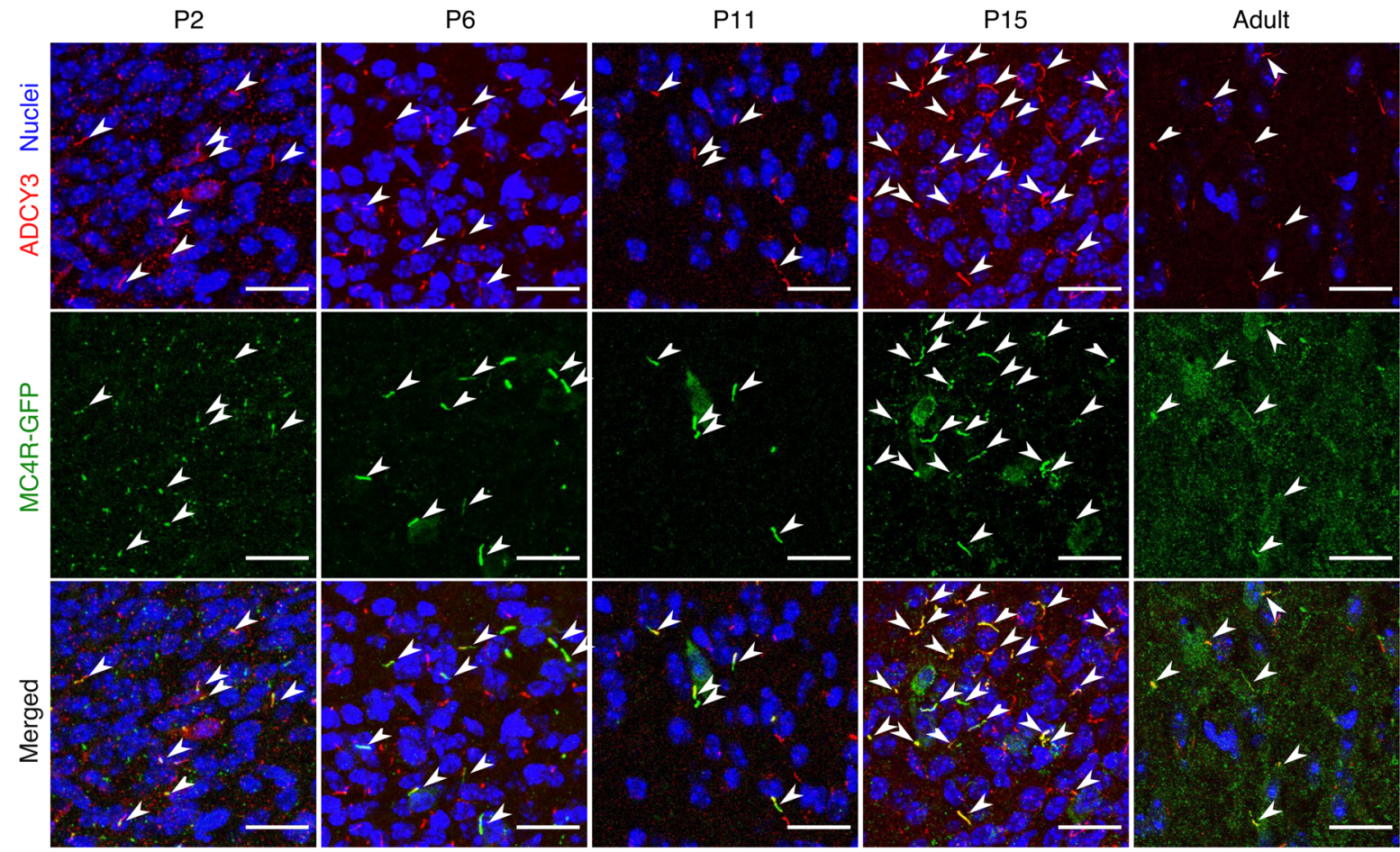

Figure 1. Developmental MC4R subcellular localization in the PVN at postnatal stages P2, P6, P11, and P15 and in adult mice. Representative immunofluorescence staining of MC4R-GFP (green) and ADCY3 (red) in brain sections from Mc4rfp/gfp mice during the postnatal period and from adult mice. Nuclei are stained with Hoescht (blue). Arrowheads indicate primary cilia expressing MC4R. A minimum of 3 mice were imaged per age. Scale bar: $20 \mu \mathrm{m}$.

\section{Results}

Genetic ablation of primary cilia in MC4R-expressing neurons phenocopies MC4R deficiency. By engineering mice in which GFP is inserted in-frame with the MC4R, we have previously shown that the MC4R colocalizes with adenylyl cyclase type 3 (ADCY3) at the neuronal primary cilia (12). This colocalization can also be observed early in postnatal development (Figure 1). We then sought to determine whether MC4R-expressing neurons require primary cilia to regulate body weight. Primary cilia can be specifically eliminated without affecting cell viability by inactivation of Ift88, a gene encoding an intraflagellar transport protein specifically required for ciliogenesis and ciliary maintenance (21). To inactivate Ift 88 in MC4R neurons, we first generated a $M c 4 r^{\text {t2acre }}$ allele by CRISPR/ Cas9-mediated zygotic recombination (Supplemental Figure 1A; supplemental material available online with this article; https:// doi.org/10.1172/JCI142064DS1). We inserted a T2A sequence and a Cre recombinase ORF at the terminator codon of $M c 4 r$ so that the endogenous promoter/enhancer elements directed Cre expression to MC4R-expressing cells. We verified the accurate insertion at the $M c 4 r$ locus by long-range PCR and sequencing. Recombinase activity, assessed in $M c 4 r^{\text {t2aCre/t2aCre }}$ Rosa26 $6^{\text {Ail4/Ail4 }}$ mice that express a red fluorescent protein in a Cre-dependent fashion, recapitulated the endogenous MC4R expression in particular in the PVN (ref. 22 and Supplemental Figure 1C). Importantly, the weight curves of mice homozygous for the insertion $\left(\mathrm{Mc}_{\mathrm{c}} \mathrm{r}^{\mathrm{t} 2 \mathrm{aCre} / \mathrm{t} 2 \mathrm{aCr}}\right)$ did not differ from those of WT littermates, indicating that the $M c 4 r^{\text {t2acre }}$ allele preserves MC4R function (Supplemental Figure 1B).

We crossed $M c 4 r^{t 2 a C r e}$ mice with Ift $88^{f / / f l}$ mice (21) to generate mice with specific deletion of Ift 88 in MC4R-expressing cells. To determine the effect of primary cilia deletion in MC4R neurons, we compared $M c 4 r^{t 2 a C r e / t 2 a C r e} I f t 88^{f / f l}$ mice with their $M c 4 r^{t 2 a C r e / t 2 a C r e}$ Ift $88^{+/+}$littermates. Mc4 $r^{t 2 a C r e / t 2 a C r e}$ Ift $88^{f / f l}$ mice were born at a Mendelian ratio and developed a severe obesity phenotype (Figure 2, A-J) that mimicked germline loss of the MC4R (18). Specifically, the body weight curves of $M c 4 r^{\text {t2aCre/t2aCre }}$ Ift $88^{f l / f l}$ and $M c 4 r^{\text {t2aCre/t2aCre }}$ Ift $88^{+/+}$mice diverged after weaning (Figure 2, A and F). This difference in body weight was characterized by a large increase in fat mass (Figure 2, B and G), as well as an increase in lean mass (Figure 2, C and H), body length (Figure 2, D and I), and food intake (Figure 2, E and J). We observed the same effect in both male (Figure 2, A-E) and female (Figure 2, F-J) mice.

To confirm that loss of primary cilia in MC4R neurons did not affect neuron survival, we generated triple-knockin $M c 4 r^{t 2 a C r e / 2 a C r e}$ Ift $88^{f l / f l}$ Rosa2 $6^{\text {Ail//Ail4 }}$ mice and found no difference in the number of MC4R-expressing neurons in the PVNs of $M c 4 r^{\text {t2aCre/t2aCre }}$ Ift $88^{f / f l}$ Rosa26 $6^{\text {Ail//Ail4 }}$ mice when compared with those of $M c 4 r^{t 2 a C r e / 2 a C r e}$ Ift88 ${ }^{+/+}$Rosa26 $6^{\text {Ail4/Ail4 }}$ control mice (Figure 2, K and L). We also sought to determine whether loss of cilia affects the capacity of MC4R neurons to decrease food intake when activated. A Cre-dependent adeno-associated virus (AAV) encoding GqDREADD-mCherry was stereotaxically injected bilaterally into the PVN of $M c 4 r^{t 2 a C r e / 2 a C r e}$ Ift $88^{f l / f l}$ and control $M c 4 r^{\text {t2aCre/t2aCre }}$ Ift $88^{+/+}$ or $I f t 88^{f l /+}$ mice. A negative control group of mice were injected with AAV DIO-mCherry (Figure 2, M-O). All 3 groups of mice were fasted for 24 hours and challenged with saline and clozapine $\mathrm{N}$-oxide (CNO) in a randomized crossover experimental design, and their 3-hour re-feeding food intake was measured (Figure 2O). Activation of MC4R neurons by CNO led to a significant 
Males
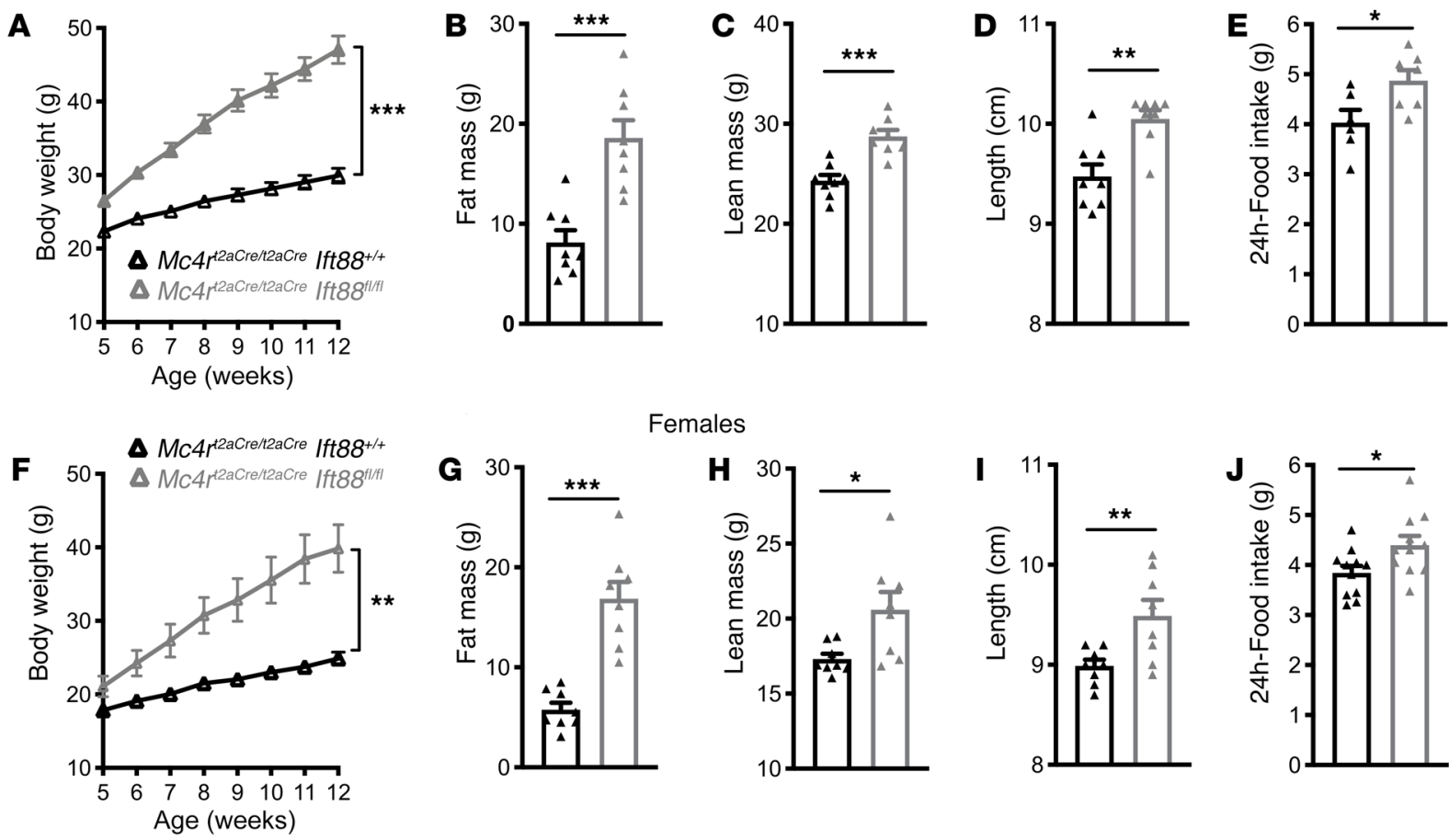

Females

$\mathbf{K}$

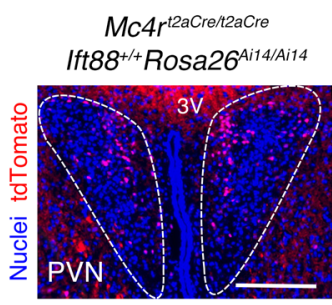

M

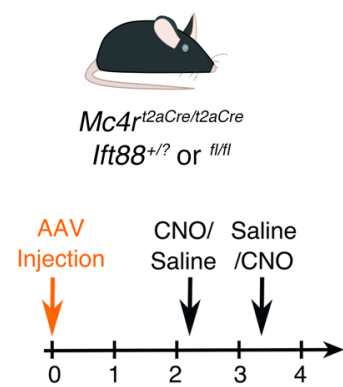

N AAV-DIO-GqDREADD or AAV DIO-mCherry (CTL) injection in the PVN

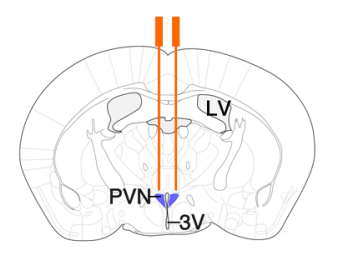

Bregma $-0.8 \mathrm{~mm}$
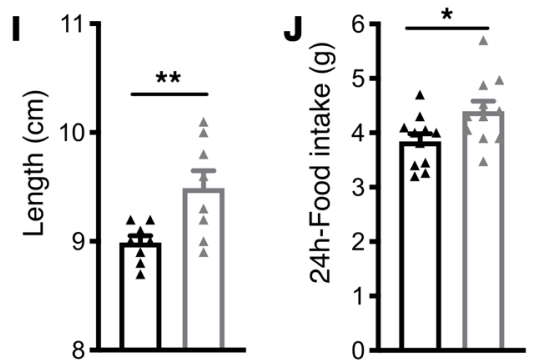
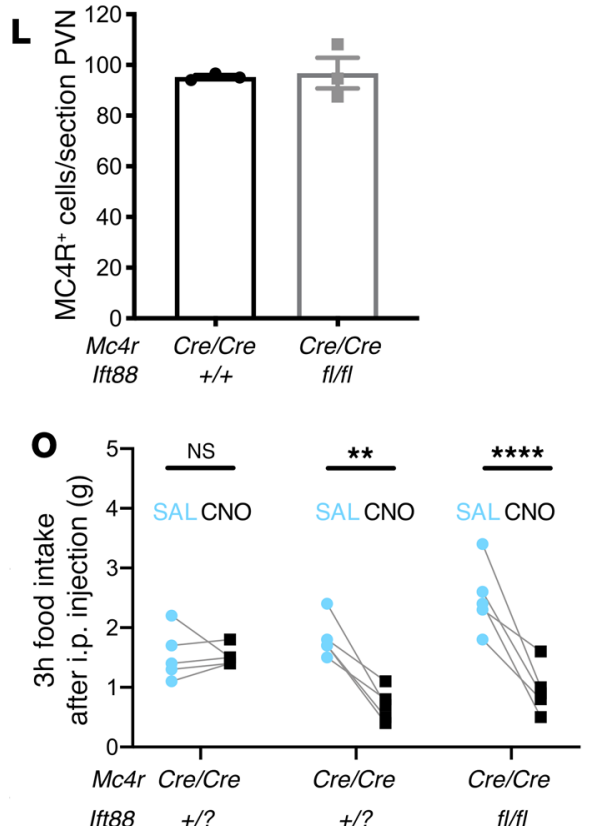

AAV CTL GqDREADD GqDREADD

Figure 2. Deletion of Ift88 in MC4R-expressing neurons leads to obesity. (A-J) Phenotyping of control Mc4R $R^{\text {tzacre/tzacre }} / f t 88^{+/+}$and $M c 4 R^{\text {tzacre/tzacre }} / f t 88^{f / / f l}$ male (A-E) and female (F-J) mice. Body weights of mice were measured weekly from 5 to 12 weeks of age (A and $\mathbf{F})$. Fat mass (B and $\mathbf{G})$ and lean mass (C and $\mathbf{H}$ ) (measured by EchoMRI) and body length ( $\mathbf{D}$ and $\mathbf{I})$ were assessed in mice at 12 weeks of age ( $n=8$ mice per group). (E and $\mathbf{J})$ Twenty-four-hour food intake at 12 weeks of age (females, $n=11$ control and $n=11$ experimental mice; males, $n=6$ control and $n=7$ experimental mice). (K) Representative imag-

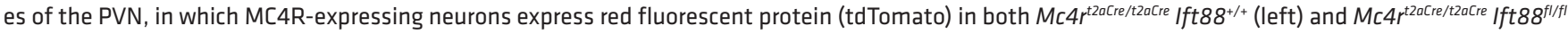

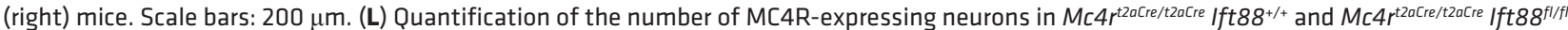

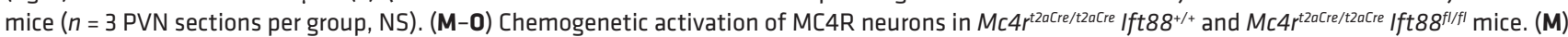
Schematic of the experimental timeline: 7- to 14-week-old male mice were stereotaxically injected with AAV DIO-GqDREADD or AAV DIO-mCherry and then tested either for CNO or saline 2 weeks and 3 weeks after injection, using a randomized crossover design. (N) Schematic of bilateral stereotaxic injections (coordinates: $A P=-0.8, M L= \pm 0.25, D V=-5.2$ ) of AAV DIO-GqDREADD or AAV DIO-mCherry as a control (CTL). (O) Three-hour food intake following injection of saline (SAL) or CNO in 3 groups of mice ( $n=5$ per group): Mc $4 r^{\text {t2acre/tzacre }} / \mathrm{ft}^{2} 88^{+/ ?}$ injected with AAV DIO-mCherry (negative control), Mc4r ${ }^{\text {t2acre/t2acre }}$ Ift8 $8^{+/ ?}$ mice injected with AAV DIO-GqDREADD (positive control), and Mc4 $\mathrm{r}^{\text {tzacre } / \text { tzacre }}$ Ift8 $88^{\mathrm{fl} / \mathrm{fl}}$ mice injected with AAV DIO-GqDREADD. Mice were fasted for 24 hours prior to injection. Data represent the mean \pm SEM. ${ }^{*} P<0.05,{ }^{* *} P<0.01,{ }^{* *} P<0.001$, and ${ }^{* * * *} P<0.0001$, by Student's $t$ test (B-E, G-J, and $\mathbf{L}$ ) or repeated-measures 2-way ANOVA followed by Sidak's multiple-comparison test (A, F, and $\mathbf{0})$. 3V, third ventricle; LV, lateral ventricle. 
decrease in food intake in both groups of mice that expressed GqDREADD, whereas the control AAV-mCherry group did not respond to CNO. This result demonstrates that loss of cilia did not impair the capacity of MC4R neurons to generate an anorexigenic response upon activation.

Together, these results indicate that primary cilia are critically and cell-autonomously required for MC4R neurons to control long-term energy homeostasis.

Primary cilia are required for anorexigenic MC4R signaling. Since loss of primary cilia did not appear to affect the survival of MC4R-expressing neurons (Figure 2, K and L) or their capacity to be artificially stimulated by DREADDs (designer receptors exclusively activated by designer drugs) (Figure 2, M-O), we hypothesized that the obesity observed in $M c 4 r^{t 2 a C r e / 2 a C r e}$ Ift $88^{f / f l}$ mice was due to impaired MC4R function in differentiated neurons. To test this hypothesis, we determined whether primary cilia are required for the anorexigenic agonist-dependent function of the MC4R in adult mice.

As opposed to ubiquitous germline ablation of primary cilia, which is embryonically lethal, ubiquitous adult conditional ablation of primary cilia, achieved by deletion of Ift 88 using a tamoxifen-inducible Cre (Ubc-Cre-Ert2 Ift $\left.88^{f / f l}\right)$, results in obesity (11).

To determine whether loss of primary cilia affects MC4R signaling, we assessed whether the timeline of primary cilia ablation and onset of the metabolic phenotype in this model is compatible with a dysfunction of the central melanocortin system. Adult $U b c$ Cre-Ert2 Ift $88^{f / f l}$ mice injected with tamoxifen were compared with tamoxifen-injected control Ift $88^{f / f l}$ mice (Figure 3A). Tamoxifen administration led to neuronal ablation of primary cilia specifically in Ubc-Cre-Ert2 Ift $88^{f / f l}$ mice within 2 weeks, as assessed by ciliary ADCY3 staining, a delay consistent with the half-life of IFT88 (Supplemental Figure 2). We assessed body weight, body composition, food intake, and energy expenditure repeatedly over a 4-week period (Figure 3, A and B). Concurrent with neuronal cilia loss, tamoxifen-injected Ubc-Cre-Ert2 Ift $88^{f / f l}$ mice developed obesity characterized by increased fat mass and a slight increase in lean mass (Figure 3, B-E). This accumulation of fat mass was associated with hyperphagia, rather than changes in energy expenditure (Figure 3, F and G), which would be consistent with loss of MC4R function in the PVN (23).

To more specifically determine whether MC4R activity was impaired following ablation of primary cilia in this model, we evaluated the anorexigenic effect of pharmacological stimulation of the MC4R before and after tamoxifen-induced ablation of primary cilia, but prior to weight divergence (Figure 4, A-C). Specifically, Ubc-Cre-Ert2 Ift $88^{f / f l}$ and control Ift $88^{f / f l}$ mice were injected i.c.v. with melanotan II (MTII), an MC4R agonist, before and after tamoxifen-mediated cilia loss (Figure 4B). Mice were fasted for 24 hours prior to i.c.v. injection, and their food intake was measured over a 4-hour period following injection. As expected, prior to tamoxifen-mediated cilia loss, we found that MTII induced a drastic reduction of food intake for mice in both groups compared with injection of the control solution, artificial cerebrospinal fluid (aCSF) (Figure 4D). After tamoxifen injection, the anorexigenic effect of MTII gradually diminished specifically in Ubc-Cre-Ert2 Ift $88^{f / f l}$ mice (Figure 4D). Thus, primary cilia are necessary for MC4R-mediated effects on food intake.
As an additional test of whether cilia are required for MC4R signaling, we assessed the effects of deleting cilia on the activity of THIQ, another specific agonist of the MC4R that inhibits food intake. Similar to MTII, the anorexigenic response to i.c.v. administration of THIQ was abolished specifically following removal of primary cilia specifically in $\mathrm{Ubc}$-Cre-Ert2 Ift $88^{f / / f l}$ mice (Supplemental Figure 3).

As observed when we specifically removed primary cilia from MC4R neurons developmentally, deletion of Ift88 from adult hypothalamus did not alter neuron numbers (Supplemental Figure 4). Deletion of Ift 88 also did not alter the expression levels of $\operatorname{Sim} 1$ or $M c 4 r$ in the adult hypothalamus (Supplemental Figure $4 \mathrm{D}$ ). These results indicate that the loss of responsiveness to MC4R stimulation upon removal of primary cilia was not due to loss of MC4R-expressing cells or decreased Mc4r expression.

In addition to regulating feeding behavior, PVN neurons respond to osmotic stimulation, for example, by phosphorylating the ribosomal protein S6 (p-S6) (24). p-S6 activation by osmotic stimulation in PVN neurons was preserved in tamoxifen-treated Ubc-Cre-Ert2 Ift $88^{f l / f l}$ mice following cilia loss, indicating that primary cilia were not required for all functions of these neurons (Supplemental Figure 4, E and F).

Adult PVN primary cilia are required for anorexigenic $M C 4 R$ signaling. The MC4R is expressed in a number of brain regions, including in multiple hypothalamic nuclei (25). However, much of the anorexigenic activity of the MC4R is due to its function in PVN neurons, where MC4R activity is both necessary and sufficient to inhibit food intake and control body weight (19, 23). In $M c 4 r^{t 2 a c r e / t 2 a C r e}$ Ift $88^{f / f l}$ mice, primary cilia were ablated from all MC4R-expressing cells, including those of the PVN. In Ubc-Cre-Ert2 Ift $88^{f / f l}$ mice, treatment with tamoxifen removed cilia globally. Therefore, to test whether primary cilia are specifically required in the PVN for the regulation of body weight through MC4R activation, we deleted Ift88 by bilateral stereotaxic injection of an AAV-expressing, GFP-tagged Cre recombinase (AAV-CreGFP) into the PVN of adult $I f t 88^{f / f l}$ mice (Figure $5 \mathrm{~A})$. Mice injected with AAV expressing a GFP-tagged functionally impaired Cre recombinase (AAV-nGFP) served as controls. GFP expression indicated infected cells and allowed for post hoc confirmation of PVN injection (Figure 5, C and D). Injection of Cre-producing virus, but not control virus, led to the ablation of primary cilia in the PVN (Figure 5, C and D, enlarged insets).

Following ablation of primary cilia in the PVN, AAV-CreGFPinjected $\mathrm{Ift} 88^{\mathrm{fl} / \mathrm{fl}}$ mice gained weight (Figure 5, E and F). Thus, cilia are critical for the regulation of energy homeostasis in the PVN and account, in full or in part, for the obesity observed after ubiquitous disruption of primary cilia in adult mice. Given that the MC4R localizes to primary cilia of neurons in the PVN (12), these data further suggest that the MC4R could function at PVN cilia to control body weight.

To determine whether MC4R activation requires PVN primary cilia, we implanted an i.c.v. cannula into the lateral ventricle at the time of AAV injection (Figure 5, G and H) and measured changes in the anorectic effect of MTII after PVN primary cilia loss as described above. Mice were injected with MTII $(0.05 \mathrm{nmol})$ or vehicle (aCSF) after a 24-hour fast, and their food intake was then measured over 4 hours. (Figure 5I). Although control AAV- 
A
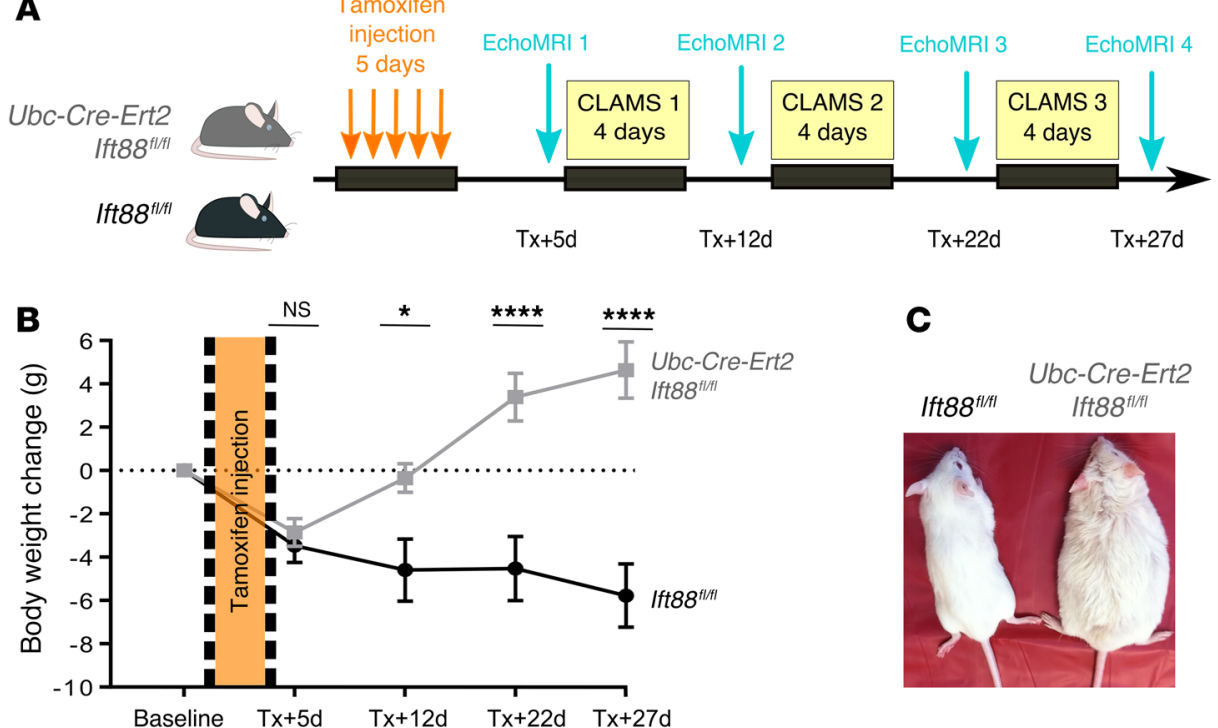

C

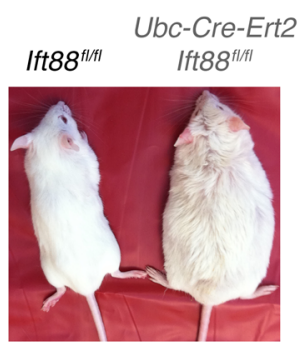

Figure 3. Time course of metabolic changes following ablation of primary cilia in adult mice. (A) Schematic of the experimental protocol. (B) Time course of body weight changes for 20-week-old male Ubc-Cre-Ert2 Ift8 $8^{f / f I}(n=8)$ and Ift8 $88^{f / f l}(n=4)$ mice at baseline and at the indicated time points after tamoxifen injection. (C) Images of male control (Ift8 $\left.8^{f / / f I}\right)$ and Ubc-Cre-Ert2 Ift $88^{f / f l}$ mice 4 weeks following tamoxifen injection. (D) Fat mass, (E) lean mass (measured by EchoMRI), (F) food intake, and (G) energy expenditure (measured by CLAMS) for Ubc-Cre-Ert2 Ift8 $8^{f / f f}$ versus Ift8 $8^{f / / f l}$ male mice at the indicated time points after tamoxifen injection. Data represent the mean \pm SEM. ${ }^{*} P<0.05,{ }^{* *} P<0.01$, and ${ }^{* * *} P<0.0001$, by 2 -way ANOVA followed by Sidak's multiple-comparison test (B and D-F). Energy expenditure in $\mathbf{G}$ was analyzed by CaIR ANCOVA, with body weight included as a covariate. Tx, tamoxifen.
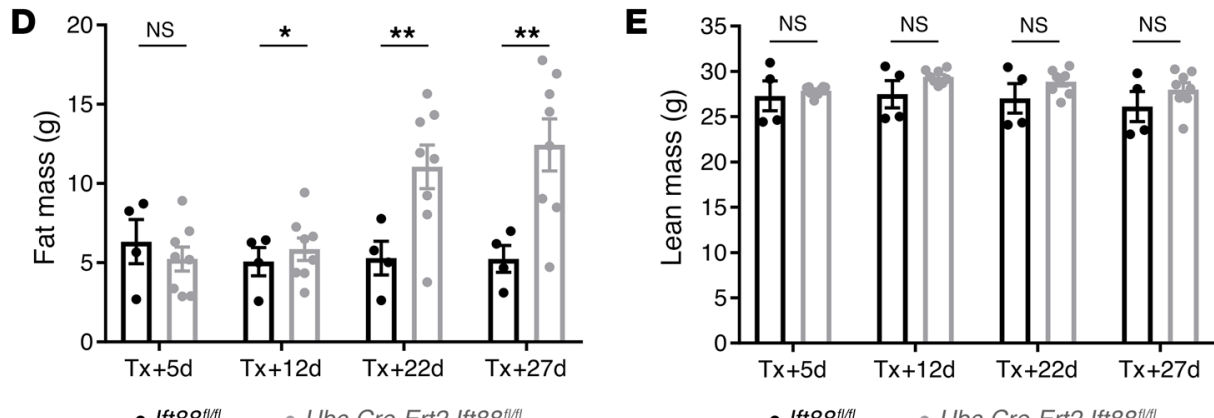

- Ift88 $8^{f / f t} \quad$ Ubc-Cre-Ert2 Ift88 fifft

\section{$\mathbf{F}$}

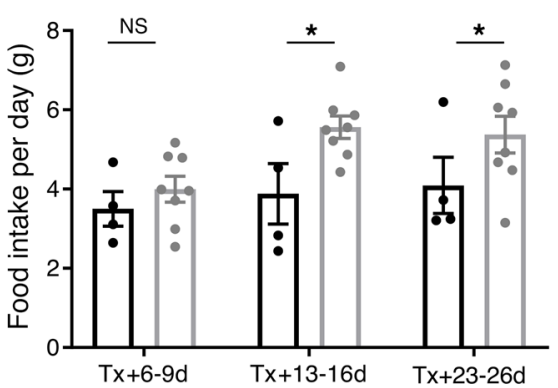

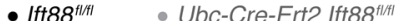

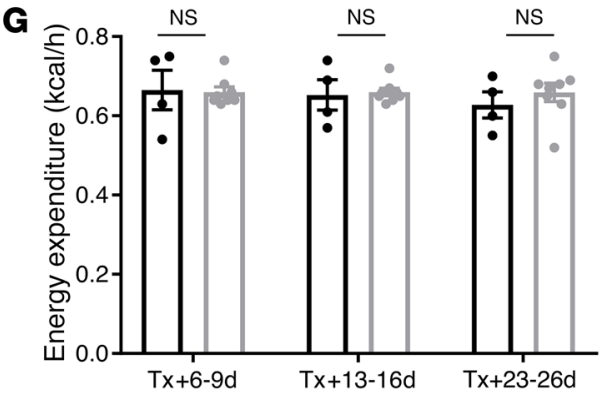

nGFP-injected mice decreased their food intake by $60 \%$ on average after MTII injection as compared with after aCSF injection, this response was blunted in AAV-CreGFP-injected mice lacking primary cilia in the PVN (Figure 5, I and J). Together, these results demonstrate that the anorexigenic function of the MC4R requires primary cilia in the PVN.

Adenylyl cyclase signaling in the primary cilia of MC4R-expressing neurons in the adult PVN is essential for controlling food intake and body weight. We reasoned that if the MC4R functions at the primary cilia of PVN neurons, then inhibition of MC4R signaling specifically in these primary cilia would increase food intake and cause obesity. Activation of the MC4R stimulates Gas to increase adenylyl cyclase (AC) activity (26). Inhibition of AC specifically at the primary cilia can be achieved by expression of a constitutively active version of the cilium-localized Gai-coupled receptor GPR88 [GPR88(G283H) or GPR88*] (27).
To target MC4R neurons of the PVN, we stereotaxically injected a Cre-dependent AAV encoding a Flag-tagged version of GPR88* (AAV DIO Flag-GPR88*) into the PVNs of 20-weekold $M c 4 r^{\text {t2acre/2acre }}$ male mice (coordinates: anteroposterior [AP] $=-0.8$, mediolateral $[\mathrm{ML}]=0.0$, dorsoventral $[\mathrm{DV}]=-5.2)$. To verify injection accuracy, PVN transduction, and Cre activity, we coinjected AAV-expressing mCherry in a Cre-dependent manner (AAV DIO-mCherry). Control mice included $M c 4 r^{\text {t2acrettzacre }}$ mice injected only with AAV DIO-mCherry as well as $\mathrm{Mc} 4 \mathrm{r}^{++}$littermates injected with AAV DIO-GPR88* and AAV DIO-mCherry (to control for Cre-independent effects of AAV DIO-GPR8 $88^{*}$ ). We measured body weights weekly and assessed body composition by EchoMRI 3, 6, and 9 weeks after the AAV injections. We used a comprehensive laboratory animal monitoring system (CLAMS) to assess food intake and energy expenditure 3 weeks after the AAV injections. 
A i.c.v. cannula

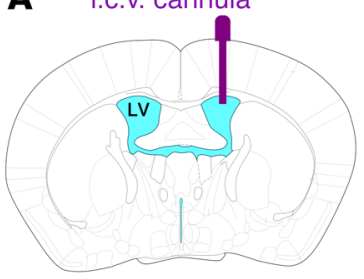

Bregma $=-0.3$
B

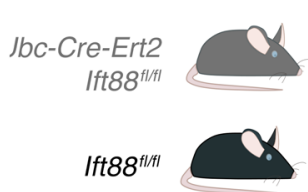

i.c.v.

cannulation

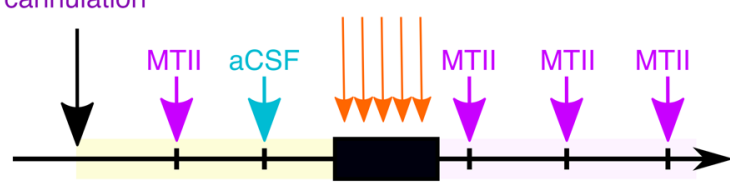

Before Tx
$T x+2 d \quad T x+11 d \quad T x+18 d$
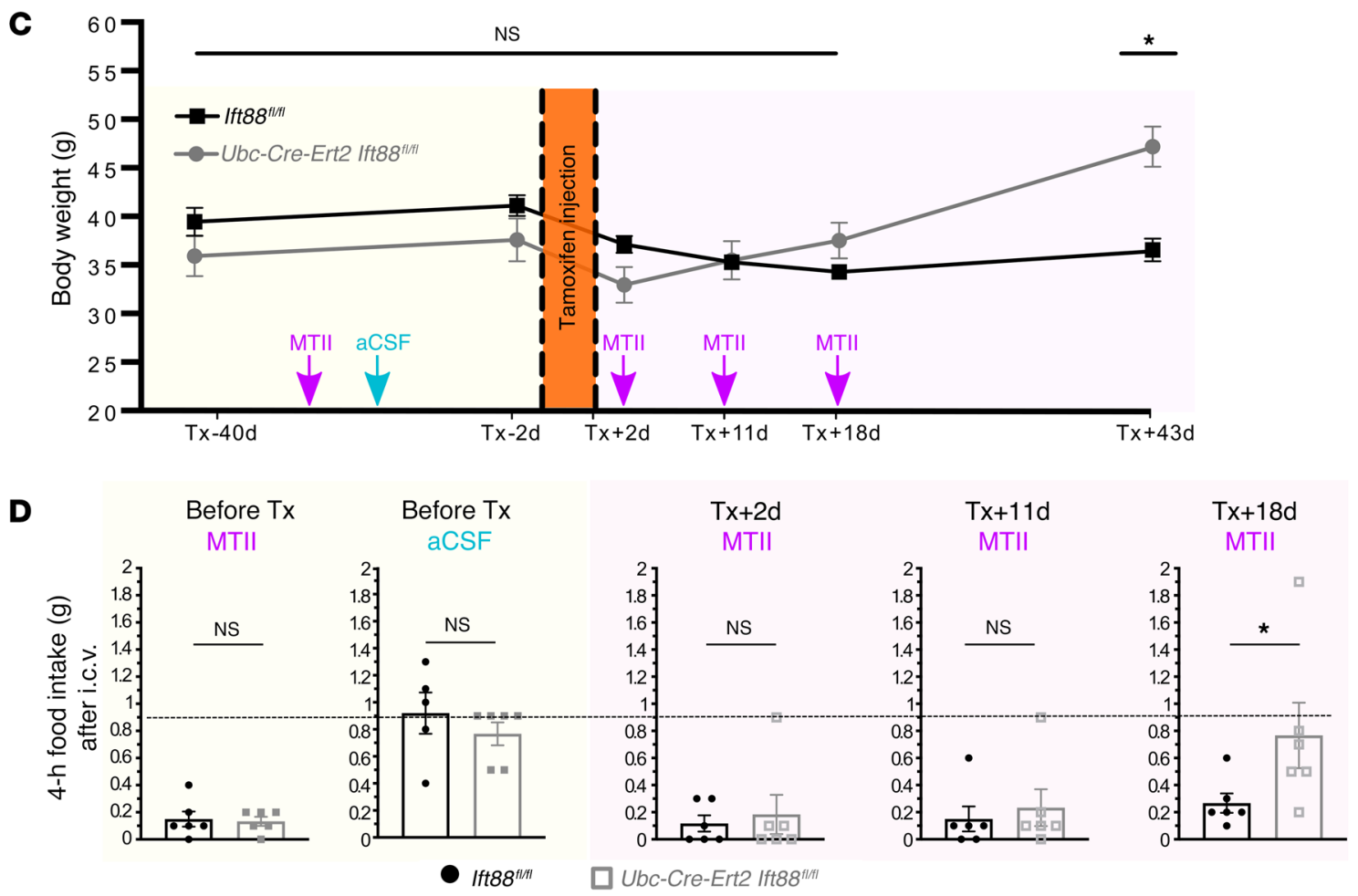

Figure 4. Primary cilia are essential for the response to the MC4R agonist MTII. (A) Schematic of the placement of an i.c.V. cannula in the lateral ventricle

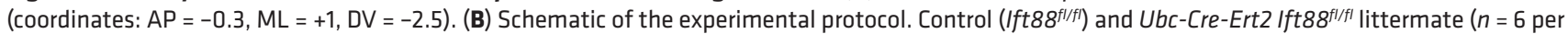
group) mice over 20 weeks of age were implanted with an i.c.v. cannula in the lateral ventricle. After recovery, food intake was measured following i.c.v. delivery of MTII or vehicle control (aCSF). Mice were then injected with tamoxifen for 5 days, and the response to i.c.v. delivered MTII was measured again 2, 11, and 18 days after the last tamoxifen injection. (C) Body weights of Ift8 $8^{f / / f l}$ control and Ubc-Cre-Ert2 Ift8 $8^{f / / f l}$ littermate mice during the experiment. (D) Assessment of the anorexigenic effect of MTII $(0.5 \mathrm{nmol})$ on short-term food intake (4 hours) compared with vehicle (aCSF) before and after primary cilia loss. Mice were fasted for 24 hours prior to injection. ${ }^{*} P<0.05$, by 2 -way ANOVA (C) and unpaired Student's $t$ test (D). Data represent the mean \pm SEM.

$M c 4 r^{t 2 a C r e / t 2 a C r e}$ mice injected with AAV DIO-GPR88* into the PVN showed increased body weight (Figure 6, E and F) and fat mass (Figure $6 \mathrm{H}$ ). We found that food intake and energy expenditure were higher in AAV DIO-GPR88*-injected $M c 4 r^{\text {t2aCret2aCre }}$ mice 3 weeks after AAV injection (Figure 6, I and J). Thus, GPR88*mediated obesity was attributable to hyperphagia.

We then determined whether MC4R activation requires $\mathrm{AC}$ signaling at the primary cilium of MC4R neurons. We implanted an i.c.v. cannula into the lateral ventricle (coordinates: $\mathrm{AP}=-0.3$, $\mathrm{ML}=+1, \mathrm{DV}=-2.5$ ) at the time of AAV injection (coordinates: AP $=-0.8, \mathrm{ML}= \pm 0.2, \mathrm{DV}=-5.2)$ and measured changes in the anorectic effect of MTII after GPR88* expression at the primary cilium of PVN MC4R neurons, as described above (Figure 6, K-M). We randomly assigned mice to 2 groups and injected them with MTII (0.5 nmoles) or vehicle (aCSF) after a 24-hour fast. Next, we measured their food intake over a 4-hour period, and the reverse treatment was completed 5 days later (Figure 6L). Although control AAV DIO-mCherry-treated mice significantly decreased their food intake after MTII injection compared with after aCSF injection, this response was blunted in mice treated with AAV

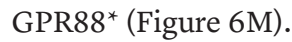

These results demonstrate that reducing AC activity specifically at the primary cilia of adult MC4R PVN neurons is sufficient to increase food intake and disrupt the regulation of body weight, and that the anorexigenic function of the MC4R requires AC signaling at the primary cilium of PVN MC4R neurons.

\section{Discussion}

Obesity is a hallmark of several human ciliopathies, including Bardet-Biedl syndrome, MORM (mental retardation, truncal obesity, retinal dystrophy, and micropenis) syndrome, and Carpenter syndrome. As the major physiological pathway implicated 
A
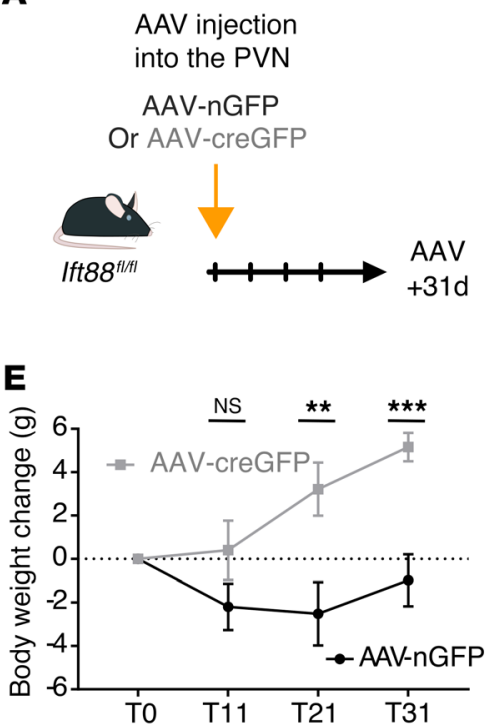

B

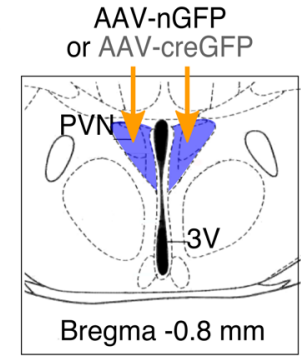

F
C

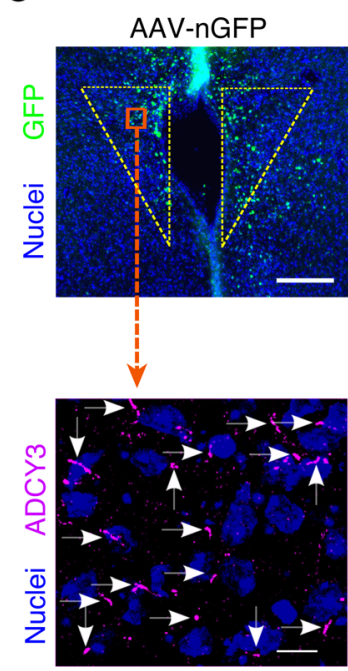

D

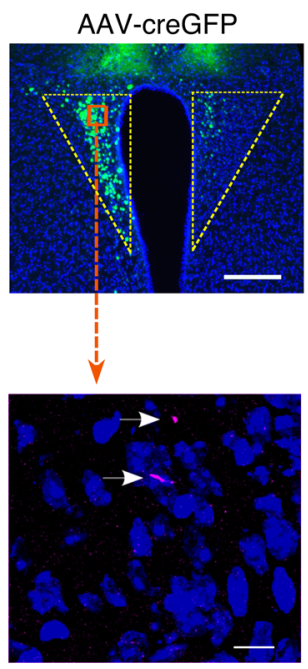

G

$$
\begin{aligned}
& \text { Acute i.c.v. cannula } \\
& \text { +AAV injection } \\
& \text { into the PVN }
\end{aligned}
$$

AAV-nGFP

Or AAV-creGFP

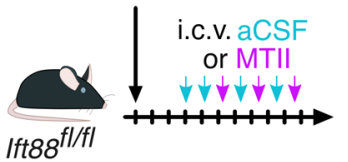

H

AAV-creGFP or -nGFP injection into the PVN

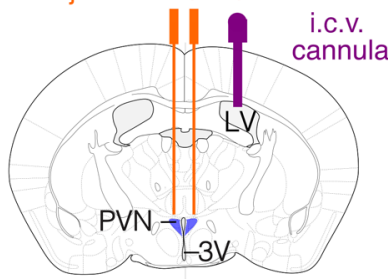

Bregma $-0.8 \mathrm{~mm}$

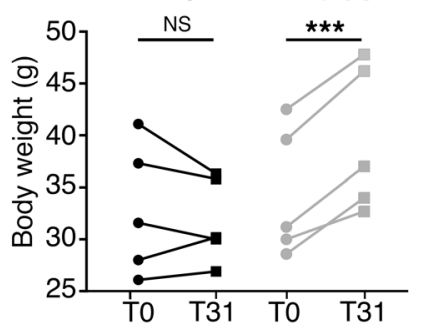

AV-creGFP

\section{I}

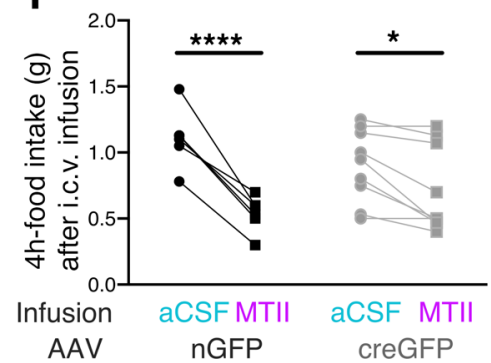

J

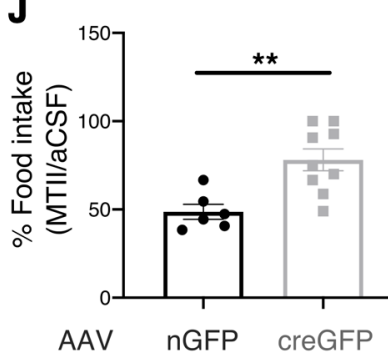

Figure 5. Primary cilia are required in PVN neurons for weight control and sensitivity to MC4R agonists. (A) Schematic of the experimental protocol. Bilateral stereotaxic injections (coordinates: $A P=-0.8, M L= \pm 0.2, D V=-5.2$ ) of AAV-CreGFP or AAV-nGFP were performed on 20-week-old Ift88 fl/fl mice. (B) Schematic representation of the hypothalamic region studied. (C and D) Representative images of PVN sections from AAV-CreGFP- or AAV-nGFPinjected mice showing AAV-infected cells in green and nuclei in blue. Scale bars: $200 \mu \mathrm{m}$. Enlarged insets show immunofluorescence images of primary cilia (ADCY3, magenta) in the PVN regions shown in $\mathbf{C}$ and $\mathbf{D}$. Arrows indicate cilia. Scale bars: $10 \mu \mathrm{m}$. (E) Body weights of Ift8 $8^{f l / f l}$ mice following bilateral PVN injection of AAV-CreGFP $(n=5)$ or AAV-nGFP $(n=5)$. (F) Body weights at the time of AAV injection and 1 month later. Individual mice are indicated by lines. (C) Schematic of the experimental protocol for testing the anorexigenic effects of the MC4R agonist MTII. Three weeks after AAV injection and cannulation, 20-week-old Ift8 $8^{\text {fl/fl }}$ mice were alternately treated with vehicle (aCSF) or MTII by i.c.v. infusion after fasting for 24 hours, with a 4-day recovery between infusions. Food intake during a 4-hour re-feeding period was then averaged for aCSF and MTII (values are shown in I and J). (H) Schematic of bilateral stereotaxic injections (coordinates: AP $=-0.8, M L= \pm 0.2, D V=-5.5$ ) of AAV-CreGFP $(n=9)$ or AAV-nGFP $(n=6)$, and placement of an i.c.v. cannula in the lateral ventricle (coordinates: $A P=-0.3, M L=+1, D V=-2.5$ ). (I) Four-hour food intake following injection of aCSF or MTII into AAV-CreGFP- and control AAV-nGFP-injected Ift88 $8^{f / f l}$ mice (repeated-measures averaged). (J) Percentage of food ingested within 4 hours following i.c.v. administration of MTII normalized to aCSF administration. Data represent the mean \pm SEM. ${ }^{*} P<0.05,{ }^{* *} P<0.01,{ }^{* * *} P<0.001$, and ${ }^{* * *} P<0.0001$, by Student's $t$ test (J) and repeated-measures 2-way ANOVA followed by Sidak's multiple-comparison test (E, F, and I).

in the regulation of long-term energy homeostasis, the central leptin-melanocortin system may link human ciliopathies and obesity. Since deletion of primary cilia in adult mice causes leptin resistance, the initial hypothesis was that dysfunction of central leptin signaling could play a role in the observed associated obesity $(11,28-30)$. However, leptin resistance was subsequently found to be a consequence, rather than a cause, of obesity in this model (31), suggesting that dysfunction of a downstream effector of leptin may account for ciliopathy-associated obesity.

Here, we used several complementary genetic and pharmacological approaches to demonstrate that the MC4R, a GPCR critically required for maintaining body weight, not only localizes to primary cilia, but also operates at the primary cilium of PVN neurons to control energy homeostasis in adult mice. Removal of primary cilia specifically from MC4R-expressing cells (in $M c 4 r^{\text {t2acreftzacre }}$ Ift $88^{\pi / f l}$ mice) phenocopied the obesity phenotype caused by germline loss of the MC4R (18), demonstrating that primary cilia are essential for the function of MC4R-expressing neurons.

As this requirement of primary cilia in MC4R-expressing neurons could reflect a developmental role for cilia caused by their removal, we investigated whether primary cilia are required for the production of MC4R-expressing neurons and found that removing cilia does not affect the number of MC4R-expressing neurons or expression of the MC4R. Furthermore, we found that MC4R neurons that had lost their cilia could still be chemogenetically activated and lead to an anorexigenic response. Therefore, primary cilia are dispensable for the production of MC4R-expressing neurons. 
A

AAV DIO mCherry (CTL)
$+/-$
AAV DIO Flag-GPR88* (GPR88)

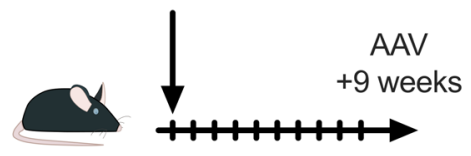

Mc4ritacrett2acre
B

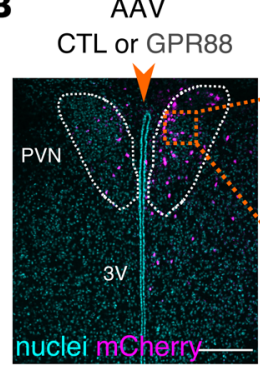

C

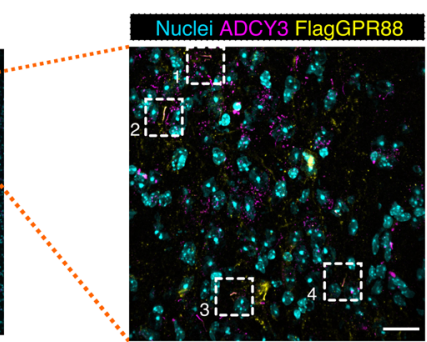

D

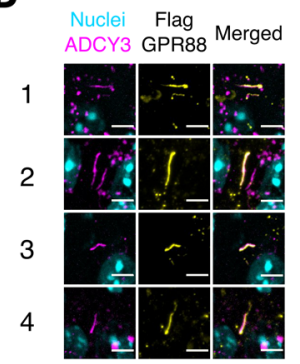

E
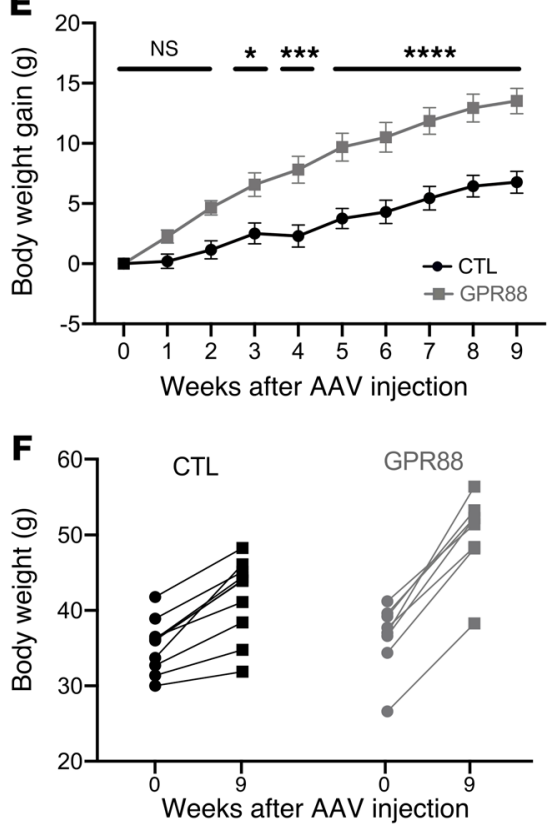

K

AAV DIO mCherry (CTL)

AAV DIO Flag-GPR88* (GPR88)

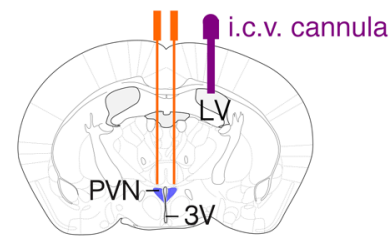

Bregma $-0.8 \mathrm{~mm}$
G

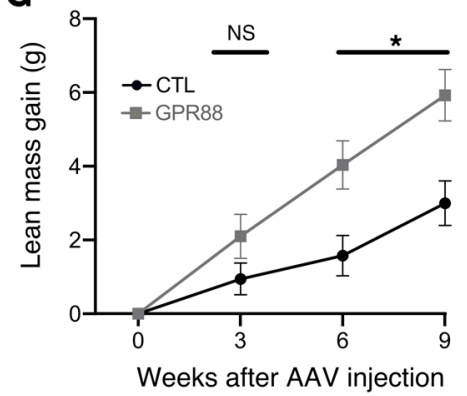

H

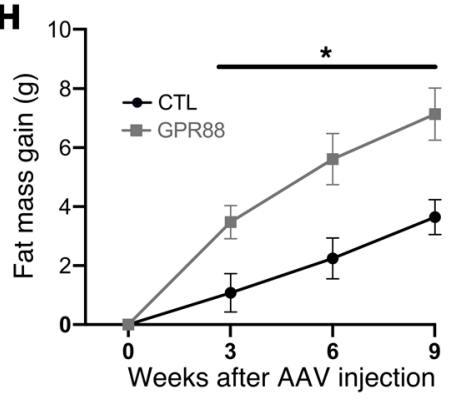

$\mathbf{L}$ i.c.V.

$+$

AAV-GPR88 or CTL

injection into the PVN

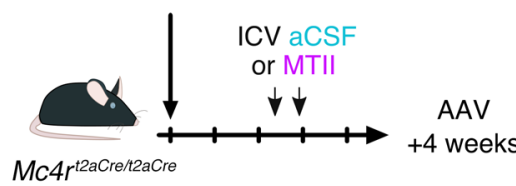

I

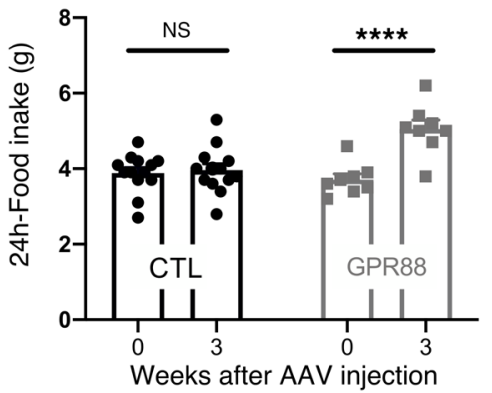

J
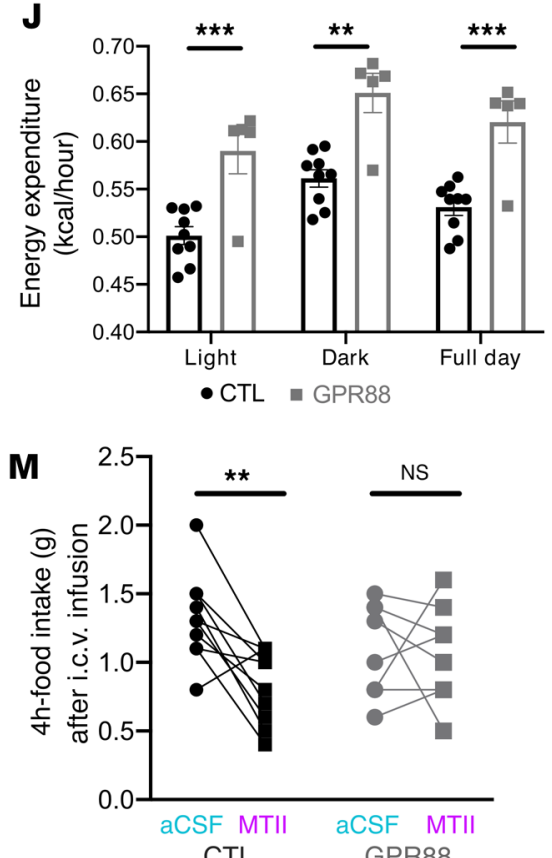

Figure 6. Inhibition of ciliary AC causes hyperphagia and obesity. (A) Schematic of the experimental protocol and midline stereotaxic injections of AAV DIO-mCherry without (CTL) or with AAV DIO-Flag-CPR88* (GPR88) into 18-week-old Mc4rtzacre/tzacre male mice. (B) Sections of PVN from GPR88-treated mice showing nuclei (cyan) and mCherry (magenta), indicating the region of viral transfection. Scale bar: $200 \mu \mathrm{m}$. (C) Enlarged immunofluorescence inset images from B showing cilia (ADCY3, magenta), Flag-GPR88* (yellow), and nuclei (cyan). Scale bar: $20 \mu \mathrm{m}$. (D) Enlarged inset images from C depicting GPR88 localization to individual cilia. Scale bars: $5 \mu \mathrm{m}$. (E) Body weight change of mice treated with GPR88 $(n=8)$ or control $(n=13)$ over a 9-week period following AAV injection. (F) Body weight at the time of AAV injection and 9 weeks later. Lines connect individual mice. Lean mass (C) and fat mass (H) gain over the 9 weeks following AAV injection. (I) Twenty-four-hour food intake at the time of AAV injection and 3 weeks later. (J) Average hourly energy expenditure during the light phase, dark phase, and full day for mice treated with GPR88 $(n=5)$ or control $(n=9)$ three weeks after AAV injection. (K) Schematic of bilateral injections of AAV DIO-mCherry $(C T L)(n=10)$ with or without AAV DIO-Flag-GPR88* $(n=8)$ or control AAV, and placement of an i.c.v. cannula in 8- to 11-week-old Mc4rtzacre/tzacre male mice. (L) Schematic of the experimental protocol for testing the anorexigenic effect of MTII. Mice were fasted for 24 hours and infused i.c.V. with vehicle (aCSF) or MTII (randomized crossover design, 2 and 3 weeks following surgery). (M) Four-hour food intake following infusion of aCSF or MTII into AAV GPR88- and control AAV-injected mice. Data represent the mean \pm SEM. ${ }^{*} P<0.05,{ }^{* *} P<0.01,{ }^{* * *} P<0.001$, and ${ }^{* * * *} P<0.0001$, by repeated-measures 2-way ANOVA followed by Sidak's multiple-comparison test (E, G-I, and $\left.\mathbf{M}\right)$. Energy expenditure in J was analyzed by ANCOVA, with body weight included as a covariate. 
Moreover, 3 additional findings demonstrated that the MC4R functions at primary cilia. First, cilia were essential for the observed anorexigenic effects of MC4R agonists such as MTII and THIQ. Second, cilia were required specifically in MC4R-expressing neurons for the regulation of feeding behavior. Third, blocking the MC4R signaling pathway by inhibiting AC specifically in the primary cilia of MC4R-expressing PVN neurons was sufficient to induce hyperphagia and obesity, as well as resistance to MTII. Since obesity was the most striking phenotype resulting from ubiquitous ablation of primary cilia in adult mice, our data suggest that a major function of adult primary cilia is to regulate long-term energy homeostasis by transducing MC4R signaling in PVN neurons.

Given that partial loss of MC4R activity, as caused by heterozygous MC4R mutations, is sufficient to cause severe obesity in mice and humans (17), a parsimonious model for ciliopathy-associated obesity is that it results from decreased localization of the MC4R to the primary cilia of PVN neurons. This model also implies that partial attenuation of ciliary localization of the MC4R is sufficient to cause obesity and that genetic variation that decreases primary cilia localization of the MC4R could predispose to weight gain. It will be interesting to determine to what extent obesity-associated variants detected in GWAS are involved in primary ciliary function. In this respect, it is interesting to note that obesity-associated variants are located within the MC4R and ADCY3 genomic regions (32) and that variants in the FTO region (the strongest obesity-associated signal in GWAS) could affect the primary cilia transition zone component RPRGRIP1L (33).

Coordinated feeding behavior results from adapting shortterm feeding behavior to immediate food availability and longterm caloric needs to maintain a stable body weight over time. Although the MC4R integrates neuroendocrine signals provided by $\alpha$-MSH and AgRP, MC4R-expressing neurons in the PVN are also sensitive to neural afferent inputs that communicate shortterm energy status from arcuate nucleus glutamatergic neurons and GABAergic AgRP-expressing neurons $(34,35)$. Our findings suggest that primary cilia could be a means for MC4R neurons to compartmentalize long-term energy state signaling resulting from MC4R activity modulation (13). This subcellular compartmentalization of signaling may explain how MC4R neurons are able to integrate different information at different timescales to coordinate feeding behavior. Future studies will address how MC4R neurons integrate ciliary and synaptic communication.

\section{Methods}

Origin of the mouse lines used. Mice were housed (with enrichment) in a barrier facility and maintained on a 12-hour light/12-hour dark cycle (on: 0700-1900 hours) at an ambient temperature of $23^{\circ} \mathrm{C} \pm$ $2{ }^{\circ} \mathrm{C}$ and relative humidity at 50\%-70\%. Mice were fed a rodent 5058 diet (LabDiet) and group housed (up to 5 mice per cage) or single housed after surgery. Experiments were performed using weightand sex-matched littermates.

Mice expressing tdTomato in a Cre-dependent manner (Gt(ROSA)

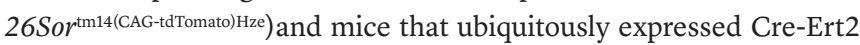
[Tg(UBC-Cre/ERT2)1Ejb, Ubc-Cre-Ert2] were obtained from The Jackson Laboratory. Mice carrying the Ift 88 conditional allele (Ift $88^{\text {tm1.1Bky, }}$ Ift $88^{f}$ ) were obtained from Bradley Yoder (University of Alabama, Bir- mingham, Alabama, USA). Experimental male Ubc-Cre-Ert2 Ift $88^{f / f l}$ mice and control Ift $88^{f / f l}$ littermates on a mixed background were obtained by crossing Ift $88^{f / f l}$ females with Ubc-Cre-Ert 2 Ift $88^{f / f l}$ males. Experimental $M c 4 r^{t 2 a C r e / t 2 a C r e} I f t 88^{f / f l}$ mice and control $M c 4 r^{t 2 a C r e / 2 a C r e}$ Ift $88^{+/+}$littermates were obtained by crossing parents that were homozygous for the $t 2 a$-Cre insertion and heterozygous for the Ift $88^{f l}$ allele.

Generation of MC4R $R^{\text {t2aCre/t2acre }}$ mice. The generation of $M C 4 R^{\text {t2aCre/t2aCre }}$ mice was performed at the Gladstone Institute mouse transgenic core as described previously for MC4R $\mathrm{R}^{\mathrm{gfp} / \mathrm{gfp}}$ mice (12). Briefly, Superovulated female FVB/N mice (4 weeks old) were mated with FVB/N stud males. Fertilized zygotes were collected from oviducts and injected with (a) Cas9 protein $(50 \mathrm{ng} / \mu \mathrm{L}),(\mathrm{b})$ a donor vector $(20 \mathrm{ng} / \mu \mathrm{L})$ consisting of $1 \mathrm{~kb}$ of the $5^{\prime}$ flanking sequence (i.e., the MC4R coding sequence) followed by t2aCre and $5.5 \mathrm{~kb}$ of the 3 ' flanking sequence, and (c) a sgRNA ( $25 \mathrm{ng} / \mu \mathrm{L}$ ), of which the guide sequence (GTCTAGCAGGTATTAAGTGG) was designed to target nucleotides immediately downstream of the MC4R stop codon in a short region that was not present in the donor vector injected into the pronucleus. Injected zygotes were implanted into oviducts of pseudopregnant CD1 female mice. Pups were genotyped for insertions at the correct loci by PCR. MC4R neuron-specific expression of Cre was verified by crossing mice

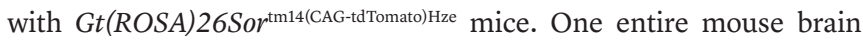
(coronal sections) was imaged and analyzed to assess Cre expression throughout the brain (Supplemental Figure 1).

Generation and injections of AAVs. AAV DJ-CMV GFP-Cre (AAVcreGFP) or AAV DJ-CMV GFP- $\triangle$ Cre (AAV-nGFP), obtained from the Stanford Neuroscience Viral Core, were stereotaxically injected bilaterally (coordinates: $\mathrm{AP}=-0.8, \mathrm{ML}= \pm 0.2, \mathrm{DV}=-5.5$, volume $=2 \times 0.5$ $\mu \mathrm{L}$ ) into the brains of Ift $88^{f / / l}$ mice. Weight was measured for 1 month, after which the mice were sacrificed to confirm the site of injection.

AAV DIO GPR88* plasmids were generated by replacing hChR2(H134R)-EYFP in pAAV-Ef1a-DIO-hChR2(H134R)-EYFPWPRE-pA (obtained from Karl Desseiroth, Stanford University, Stanford, California, USA) with GPR88(G283H)-FLAG. AAV DJ was prepared and titrated by the Stanford Neuroscience Viral Core which also provided the stock mCherry DIO-AAV (GVVC-AAV-14).

AAV DIO mCherry $(0.2 \mu \mathrm{L})$ with or without DIO GPR88 $(0.8 \mu \mathrm{L})$ was stereotaxically injected into the PVN (coordinates: $\mathrm{AP}=-0.8, \mathrm{ML}$ $=0.0, \mathrm{DV}=-5.2)$ of $M c 4 r^{\text {t2acre/t2acre }}$ mice to anatomically and genetically restrict expression to PVN MC4R-expressing neurons. Controls were either WT mice injected with DIO-GPR88 plus DIO-mCherry AAV or $M c 4 r^{\text {t2acre/t2acre }}$ mice injected solely with AAV DIO-mCherry $(0.2 \mu \mathrm{L})$ diluted in aCSF $(0.8 \mu \mathrm{L})$.

AAV DJ-hSyn-DIO-hM3Dgq-mCherry (AAV-GqDREADD), obtained from the Stanford Neuroscience Viral Core (AAV142), was stereotaxically injected bilaterally (coordinates: $\mathrm{AP}=-0.8, \mathrm{ML}=$ $\pm 0.25, \mathrm{DV}=-5.2$, volume $=2 \times 0.5 \mu \mathrm{L})$ into the PVN of $M c 4 r^{\text {t2acre } / 2 a \mathrm{Cre} e}$ Ift $88^{f / f l}$ or control $\left(\mathrm{Mc}^{\mathrm{f}} \mathrm{r}^{\mathrm{t} 2 a \mathrm{Cr} / \mathrm{t} 2 \mathrm{aCre}} \mathrm{Ift} 88^{\mathrm{fl} /+}\right.$ or Ift $\left.88^{+/+}\right)$mice. The negative control group mice ( $M c 4 r^{\text {t2aCre/t2aCre }}$ Ift $88^{\mathrm{fl} /++}$ or Ift $\left.88^{+/+}\right)$were injected with DIO-mCherry AAV.

Stereotaxic surgeries. Animals were anesthetized with an initial flow of $4 \%$ isoflurane, maintained under anesthesia using $2 \%$ isoflurane, and kept at $30^{\circ} \mathrm{C}$ to $37^{\circ} \mathrm{C}$ using a custom heating pad. The surgery was performed using aseptic and stereotaxic techniques. Briefly, the animals were put into a stereotaxic frame (KOPF Model 1900), the scalp was opened, the planarity of the skull was adjusted, and holes were drilled (coordinates: $\mathrm{PVN}, \mathrm{AP}=-0.8, \mathrm{ML}= \pm 0.2, \mathrm{DV}$ 
$=-5.5$; lateral ventricle for cannula implantation, $\mathrm{AP}=-0.3, \mathrm{ML}=1.0$, $\mathrm{DV}=-2.5)$. The AAVs were injected at a rate of $0.1 \mu \mathrm{L} / \mathrm{min}$, and guide cannulas (PlasticsOne, $2.5 \mathrm{~mm}$ ) were implanted and secured to the skull using a tissue bonding glue (Loctite 454) and dental acrylic, and then closed with a screw-on dummy cannula. Animals were given preoperative analgesic (buprenorphine, $0.3 \mathrm{mg} / \mathrm{kg}$ ) and the postoperative antiinflammatory meloxicam $(5 \mathrm{mg} / \mathrm{kg})$ and allowed to recover for at least 10 days, during which time they were single housed and handled frequently.

Mouse metabolism studies. Mice were single housed after AAV injection. Weight was measured weekly, or as mentioned in the figures. Fat mass and lean mass were measured using the EchoMRI system. Food intake was assessed either by hand or measured by CLAMS (Columbus Instruments). Energy expenditure was measured by CLAMS. Mice were tested over 96 continuous hours, and the data from the last 48 hours were analyzed. Energy expenditure (EE) is expressed in terms of kilocalories per hour and calculated using the Lusk equation: $\mathrm{EE}=$ $(3.815+1.232 \times$ respiratory exchange ratio $[\mathrm{RER}]) \times$ oxygen consumption rate $\left(\mathrm{VO}_{2}\right)$, and was analyzed with CalR software (36) (ANCOVA with body weight used as a covariate).

Specifically for Figure 2, EchoMRI was performed in mice at 12 weeks of age, and 24-hour food intake was measured manually daily over a 4 -day period. For Figure 3, EchoMRI was performed on days $+5,+12,+22$, and +27 after tamoxifen injection, and food intake and energy expenditure were measured by CLAMS on days $+6-9,+13-16$, and +23-36 after tamoxifen injection. For Figure 6, EchoMRI was performed at $t 0$ and 3, 6, and 9 weeks after AAV injection, and food intake and energy expenditure were measured by CLAMS 3 weeks after AAV injection.

Acute food intake studies. Experiments to measure the anorexigenic response to MTII were performed as described previously (37). Mice were deprived of food for 24 hours and injected i.c.v. with either $1 \mu \mathrm{L}$ aCSF or MTII (Genescript) in $1 \mu \mathrm{L}$ aCSF over a 15 -second period. For Figure 4, MTII (0.5 nmoles) was injected on days $-2,+2,+11$, and +18 after tamoxifen injection, prior to weight divergence. In the experiment depicted in Figure 5, MTII (0.05 nmoles) was injected, and for the experiment described in Figure 6, 0.5 nmoles MTII was injected (randomized crossover study). Each mouse was returned to its home cage and a weighed amount of chow was placed into the cage. The remaining chow was weighed after 4 hours to determine intake. Permeability and placement of the cannula were assessed before and after the protocol by measuring the drinking response to angiotensin II.

Experiments to determine the anorexigenic response to THIQ treatment were performed as described previously (38). Mice were deprived of food at $5 \mathrm{pm}$, injected with $1 \mu \mathrm{L}$ aCSF or $1 \mu \mathrm{L}(32 \mathrm{nM})$ THIQ (Tocris Bioscience) in aCSF at $7 \mathrm{pm}$, and food intake was measured 4 hours later.

Chemogenetic studies. CNO was administered i.p. at $2 \mathrm{mg} / \mathrm{kg}$ body weight. Saline was delivered at the same volume and at the same concentration of DMSO (2\%). Mice were fasted for 24 hours prior to injection and given access to food 30 minutes after the injection. Food intake was measured as describe above 3 hours later.

Direct fluorescence and immunofluorescence studies of mouse hypothalamus. Mice were perfused transcardially with PBS followed by $4 \%$ paraformaldehyde fixation solution. Brains were dissected and postfixed in fixation solution at $4^{\circ} \mathrm{C}$ overnight, soaked in $30 \%$ sucrose solution overnight, embedded in O.C.T. (Tissue-Tek, Sakura Finetek
USA), frozen, and then cut into $20-35 \mu \mathrm{m}$ coronal sections and stored at $-80^{\circ} \mathrm{C}$ until use for staining.

After washing, sections were blocked for 1 hour in $50 \%$ serum, $50 \%$ antibody buffer $(1.125 \% \mathrm{NaCl}, 0.75 \%$ Tris base, $1 \%$ BSA, $1.8 \%$ L-lysine, $0.04 \%$ azide), followed by incubation with primary antibodies overnight at $4^{\circ} \mathrm{C}$. After washing, the sections were incubated with secondary antibodies for 1 hour at room temperature, washed and stained with Hoechst (1:5000), and then washed and mounted with ProLong Diamond Antifade Mountant (Thermo Fisher Scientific). The following primary antibodies were used: chicken anti-GFP (Abcam, ab13970, 1:250); rabbit anti-Adcy3 (Santa Cruz Biotechnology, sc-588, 1:500); mouse anti-Flag M2 (MilliporeSigma, F1804, 1:500); and rabbit anti-p-S6 ribosomal protein (Invitrogen, Thermo Fisher Scientific, 44-923-G, 1:1000).

The following secondary antibodies were used: goat anti-chicken Alexa Fluor 488 (Invitrogen, Thermo Fisher Scientific, A11039), 1:500; goat anti-rabbit Alexa Fluor 633 or 555 or 488 (Invitrogen, Thermo Fisher Scientific, A21070, A21429, A11034), 1:500; goat anti-mouse Alexa Fluor 555 (Invitrogen, Thermo Fisher Scientific, A21424), 1:500.

RNAScope. To detect single mRNA molecules, RNAScope (Advanced Cell Diagnostics [ACD]) was performed on $20 \mu \mathrm{m}$ frozen hypothalamic sections from a P6 $\mathrm{Mc}^{\mathrm{r}} \mathrm{r}^{\mathrm{t2aCre} / 2 a \mathrm{Cre}}$ pup brain prepared as described above. The RNAScope Fluorescent Multiplex Kit V2 (cata$\log 323100$ ) was used according to the manufacturer's instructions. Briefly, slides were dried at $-20^{\circ} \mathrm{C}$ for 1 hour and then at $-60^{\circ} \mathrm{C}$ for 30 minutes. Next, they were postfixed in $4 \%$ paraformaldehyde before being dehydrated in 50\%, 70\%, and $100 \%$ ethanol for 5 minutes. After letting the slides dry for 5 minutes at room temperature, hydrogen peroxide was added to the slides for 8 minutes. Target retrieval was performed in a steamer at $100^{\circ} \mathrm{C}$. Slides were first equilibrated to the steamer in distilled water and then placed in ACD's $1 \times$ target retrieval agent. Slides were then allowed to dry overnight. The next day, the slides were treated with protease III for 10 minutes at room temperature. Cre-C2 (ACD, 312281-C2) and Mc4r-C3 (ACD, 319181$C 3)$ probes were diluted at a 1:50 ratio with probe diluent (ACD, 300041) and incubated on the slides for 2 hours at $40^{\circ} \mathrm{C}$, followed by 3 amplification steps. Opal 520 (Akoya Biosciences, FP1487001KT) and Opal 570 (Akoya Biosciences, FP148800KT) fluorophores were diluted 1:1500 in TSA buffer. The Cre-C2 probe was developed with Opal 520 Fluorophore, and the Mc4r-C3 probe was developed with Opal 570 Fluorophore. DAPI was added to label the nuclei, and slides were mounted with ProLong Gold (Thermo Fisher Scientific).

Image capture and processing. Confocal images were generated using a Leica SP5 (Supplemental Figures 2 and 4), a Zeiss LSM 780 confocal microscope (Figure 5), or a Nikon W1 spinning disk confocal microscope (Figure 6). RNAScope images (Supplemental Figure 1) were acquired with a Leica SP8 confocal microscope.

Images were processed with Fiji software (ImageJ, NIH). Maximal intensity Z projections are from at least 20 slices over $10-20 \mu \mathrm{m}$ in size. Wide-field images of p-S6 staining (Supplemental Figure 4) were generated using a Zeiss Apotome. Quantification of $\mathrm{p}-\mathrm{S6}^{+}$cells was performed with Fiji.

Tamoxifen-induced recombination. Ubc-Cre-Ert2-dependent recombination was induced by i.p. administration of tamoxifen (MilliporeSigma) for 5 consecutive days at a dose of $10.0 \mathrm{mg} / 40 \mathrm{~g}$ body weight.

Osmotic stimulation. Mice were given an i.p. injection of $2 \mathrm{M} \mathrm{NaCl}$ $(350 \mu \mathrm{L})$ or normal saline $(0.9 \% \mathrm{NaCl}, 350 \mu \mathrm{L})$ as a control. Water was 
removed from the cage, and mice were allowed access to food and were perfused 120 minutes later.

Hypothalamic RNA and protein quantification. Hypothalamic tissue was collected by dissection. IFT88 protein levels were assayed by immunoblotting as previously described (39). The following primary antibodies were used: rabbit anti-IFT88 (Proteintech, 13967-1AP, 1:500) and mouse anti-GAPDH (6C5, Santa Cruz Biotechnology, 1:1000). The following secondary antibodies were used: goat antirabbit IgG-HRP (Santa Cruz Biotechnology, 1:3000) and goat antimouse IgG-HRP (Santa Cruz Biotechnology, 1:3000). mRNA levels were assayed by quantitative RT-PCR using TaqMan assays (Thermo Fisher Scientific) for Ift88 (Mm01313467_m1), Sim1 (Mm00441391_ m1), Mc4r (Mm00457483_s1), and Actb (Mm00607939_s1).

Statistics. Sample sizes were chosen on the basis of the estimated effect size drawn from previous publications (12) and from the experiments performed in this study. Data distribution was assumed to be normal, but this was not formally tested. Statistical analysis was performed using an unpaired Student's $t$ test or repeated-measures 2-way ANOVA followed by Sidak's multiple-comparison test. All data are expressed as the mean \pm SEM. A $P$ value of 0.05 or less was considered statistically significant. Data were analyzed using Prism 8.0 (GraphPad Software) or CalR software for energy expenditure (ANCOVA with body weight included as a covariate, ref. 36).
Study approval. All animal procedures were approved by the IACYC of UCSF.

\section{Author contributions}

$\mathrm{YW}, \mathrm{AB}, \mathrm{FC}, \mathrm{JFR}$, and $\mathrm{CV}$ designed the research study and wrote the manuscript. $\mathrm{YW}, \mathrm{AB}, \mathrm{FC}$, and $\mathrm{CV}$ conducted experiments and acquired and analyzed data. XY, CP, and SZ conducted experiments.

\section{Acknowledgments}

This research was supported by NIH grants R01AR05439 and R01GM095941 (toJFR), R01DK60450 (to CV), and R01DK106404 (to JFR and CV); Sandler Integrative Research Award (to CV and JFR); and a fellowship award from the Société Francophone du Diabète (to FC). Experiments in this study were performed at the UCSF NORC Mouse Metabolism and Genetics cores, which are supported by the UCSF NORC (NIH P3ODK098722) and at the Biological Imaging Development CoLab (BIDC), which is supported by the UCSF DRC (NIH P30 DK063720).

Address correspondence to: Christian Vaisse, University of California San Francisco, 513, Parnassus Avenue, Room HSW1113, San Francisco, California 94143-0540, USA. Phone: 415. 514.0530; Email: vaisse@medicine.ucsf.edu.
1. Singla V, Reiter JF. The primary cilium as the cell's antenna: signaling at a sensory organelle. Science. 2006;313(5787):629-633.

2. Reiter JF, Leroux MR. Genes and molecular pathways underpinning ciliopathies. Nat Rev Mol Cell Biol. 2017;18(9):533-547.

3. Saeed S, et al. Loss-of-function mutations in ADCY3 cause monogenic severe obesity. Nat Genet. 2018;50(2):175-179.

4. Shalata A, et al. Morbid obesity resulting from inactivation of the ciliary protein CEP19 in humans and mice. Am J Hum Genet. 2013;93(6):1061-1071.

5. Wang $\mathrm{Z}$, et al. Adult type 3 adenylyl cyclase-deficient mice are obese. PLoS One. 2009;4(9):e6979.

6. Acs $\mathrm{P}$, et al. A novel form of ciliopathy underlies hyperphagia and obesity in Ankrd26 knockout mice. Brain Struct Funct. 2015;220(3):1511-1528.

7. Vaisse C, et al. Cilia and obesity. Cold Spring Harb Perspect Biol. 2017;9(7):a028217.

8. Hildebrandt F, et al. Ciliopathies. N Engl J Med. 2011;364(16):1533-1543.

9. Wong SY, Reiter JF. The primary cilium at the crossroads of mammalian hedgehog signaling. Curr Top Dev Biol. 2008;85:225-260.

10. Bangs F, Anderson KV. Primary cilia and mammalian hedgehog signaling. Cold Spring Harb Perspect Biol. 2017;9(5):a028175.

11. Davenport J, et al. Disruption of intraflagellar transport in adult mice leads to obesity and slow-onset cystic kidney disease. Curr Biol. 2007;17(18):1586-1594.

12. Siljee JE, et al. Subcellular localization of MC4R with ADCY3 at neuronal primary cilia underlies a common pathway for genetic predisposition to obesity. Nat Genet. 2018;50(2):180-185.

13. Krashes MJ, et al. Melanocortin-4 receptor-reg- ulated energy homeostasis. Nat Neurosci. 2016;19(2):206-219.

14. Loos RJF, et al. Common variants near MC4R are associated with fat mass, weight and risk of obesity. Nat Genet. 2008;40(6):768-775.

15. Lubrano-Berthelier C, et al. Melanocortin 4 receptor mutations in a large cohort of severely obese adults: prevalence, functional classification, genotype-phenotype relationship, and lack of association with binge eating. JClin Endocrinol Metab. 2006;91(5):1811-1818.

16. Vaisse C, et al. A frameshift mutation in human MC4R is associated with a dominant form of obesity. Nat Genet. 1998;20(2):113-114.

17. Vaisse $\mathrm{C}$, et al. Melanocortin-4 receptor mutations are a frequent and heterogeneous cause of morbid obesity. J Clin Invest. 2000;106(2):253-262.

18. Huszar D, et al. Targeted disruption of the melanocortin-4 receptor results in obesity in mice. Cell. 1997;88(1):131-141.

19. Shah BP, et al. MC4R-expressing glutamatergic neurons in the paraventricular hypothalamus regulate feeding and are synaptically connected to the parabrachial nucleus. Proc Natl Acad Sci U S A. 2014;111(36):13193-13198.

20. Pan WW, Myers MG. Leptin and the maintenance of elevated body weight. Nat Rev Neurosci. 2018;19(2):95-105.

21. Haycraft CJ, et al. Intraflagellar transport is essential for endochondral bone formation. Development. 2007;134(2):307-316.

22. Garfield AS, et al. A neural basis for melanocortin-4 receptor-regulated appetite. Nat Neurosci. 2015;18(6):863-871.

23. Balthasar N, et al. Divergence of melanocortin pathways in the control of food intake and energy expenditure. Cell. 2005;123(3):493-505.

24. Knight ZA, et al. Molecular profiling of activated neurons by phosphorylated ribosome capture. Cell. 2012;151(5):1126-1137.

25. Kishi T, et al. Expression of melanocortin 4 receptor mRNA in the central nervous system of the rat. J Comp Neurol. 2003;457(3):213-235.

26. Ersoy BA, et al. Mechanism of N-terminal modulation of activity at the melanocortin-4 receptor GPCR. Nat Chem Biol. 2012;8(8):725-730.

27. Marley A, et al. GPR88 reveals a discrete function of primary cilia as selective insulators of GPCR cross-talk. PLoS One. 2013;8(8):e70857.

28. Guo D-F, et al. The BBSome controls energy homeostasis by mediating the transport of the leptin receptor to the plasma membrane. PLoS Genet. 2016;12(2):e1005890.

29. Rahmouni K, et al. Leptin resistance contributes to obesity and hypertension in mouse models of Bardet-Biedl syndrome. J Clin Invest. 2008;118(4):1458-1467.

30. Seo S, et al. Requirement of Bardet-Biedl syndrome proteins for leptin receptor signaling. Hum Mol Genet. 2009;18(7):1323-1331.

31. Berbari NF, et al. Leptin resistance is a secondary consequence of the obesity in ciliopathy mutant mice. Proc Natl Acad Sci US A. 2013;110(19):7796-7801.

32. Ghosh S, Bouchard C. Convergence between biological, behavioural and genetic determinants of obesity. Nat Rev Genet. 2017;18(12):731-748.

33. Stratigopoulos G, et al. Hypomorphism of Fto and Rpgrip1l causes obesity in mice. JClin Invest. 2016;126(5):1897-1910.

34. Chen Y, et al. Sustained NPY signaling enables AgRP neurons to drive feeding. Elife. 2019;8:e46348.

35. Krashes MJ, et al. Rapid versus delayed stimulation of feeding by the endogenously released AgRP neuron mediators GABA, NPY, and AgRP. 
Cell Metab. 2013;18(4):588-595.

36. Mina AI, et al. CalR: a web-based analysis tool for indirect calorimetry experiments. Cell Metab. 2018;28(4):656-666.

37. Rowland NE, et al. Effect of MTII on food intake and brain c-Fos in melanocortin-3, melanocortin-4, and double MC3 and MC4 receptor knockout mice. Peptides. 2010;31(12):2314-2317.

38. Cepoi D, et al. Assessment of a small molecule melanocortin-4 receptor-specific agonist on ener- gy homeostasis. Brain Res. 2004;1000(1-2):64-71. 39. Reed AS, et al. Functional role of suppressor of cytokine signaling 3 upregulation in hypothalamic leptin resistance and long-term energy homeostasis. Diabetes. 2010;59(4):894-906. 